\title{
Sporadic Adult-onset Hypophosphatemic Osteomalacia Caused by Excessive Action of Fibroblast Growth Factor 23
}

\author{
Chisho Hoshino, Noriyuki Satoh, Shinichi Sugawara, Chizuko Kuriyama, \\ Akio Kikuchi and Masahiro Ohta
}

\begin{abstract}
A 50-year-old man without family history of metabolic bone disease was referred to our hospital with a 5year history of progressively worsening spinal and bilateral diffuse leg pain and proximal muscle weakness. Two years before admission, he was diagnosed as ankylosing spondylitis by a rheumatologist and was maintained on low-dose prednisone therapy without benefit. He developed progressive spinal and thoracic deformities, resulting in a $10 \mathrm{~cm}$ loss in height in the preceding 2 years. On physical examination, marked thoracic kyphosis and pectus carinatum was noted. Plain radiograph revealed pseudofracture in the right femoral neck. Laboratory findings showed a normal level of serum calcium, elevated level of serum alkaline phosphatase and inappropriately increased urinary phosphate excretion despite extreme hypophosphatemia. $\mathrm{He}$ was diagnosed as adult-onset hypophosphatemic osteomalacia caused by renal phosphate wasting. Serum fibroblast growth factor 23 was the upper limit of normal despite extreme hypophosphatemia and no neoplastic lesion potentially inducing hypophosphatemic osteomalacia could be identified in a thorough search including imaging studies of his entire body. Oral administration of phosphate and activated vitamin D together with dipyridamole relieved the persistent pain and weakness, and he became fully ambulatory.
\end{abstract}

Key words: osteomalacia, hypophosphotemia, adult onset disorder, fibroblast growth factor 23 , tumor induced disorder, dipyridamole

(Inter Med 47: 453-457, 2008)

(DOI: 10.2169/internalmedicine.47.0665)

\section{Introduction}

The major hereditary causes of hypophosphatemic osteomalacia associated with fibroblast growth factor 23 (FGF23) are X-linked hypophosphatemic rickets/osteomalacia (XLH), autosomal dominant hypophosphatemic rickets/osteomalacia (ADHR) and autosomal recessive hypophosphatemic rickets/ osteomalacia (ARHR). In acquired cases, tumor-induced osteomalacia (TIO) is the most representative disorder. Since these disorders have similar clinical manifestations, differentiation between these conditions is often difficult in adultonset cases. However, diagnosis of TIO is therapeutically beneficial because clinical manifestations are expected to improve after the removal of the responsible tumor (1).

Here, we report a case of sporadic adult-onset hypophosphatemic osteomalacia caused by excessive action of
FGF23, a highly suspected TIO. The clinical manifestations improved as a result of long-term oral administration of phosphate and activated vitamin $\mathrm{D}$ together with dipyridamole.

\section{Case Report}

A 50-year-old man was referred to our hospital with a 5year history of progressively worsening spinal and bilateral diffuse leg pain and proximal muscle weakness. Two years before admission, he was diagnosed as ankylosing spondylitis by a rheumatologist and was maintained on low dose prednisone therapy without benefit. He developed progressive spinal and thoracic deformities, resulting in a $10 \mathrm{~cm}$ loss in height, and had become unable to walk owing to the diffuse leg pain and proximal muscle weakness in the preceding 2 years. 

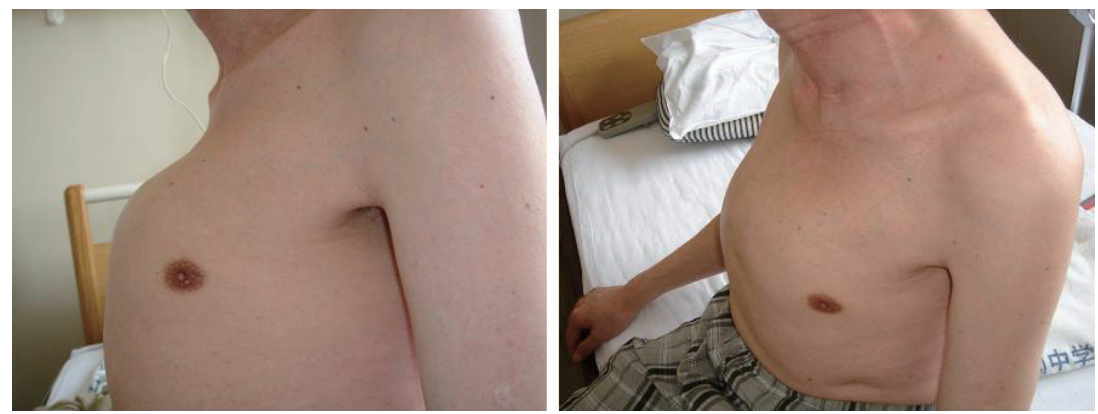

Figure 1. The patient's upper trunk presented with marked pectus carinatum.

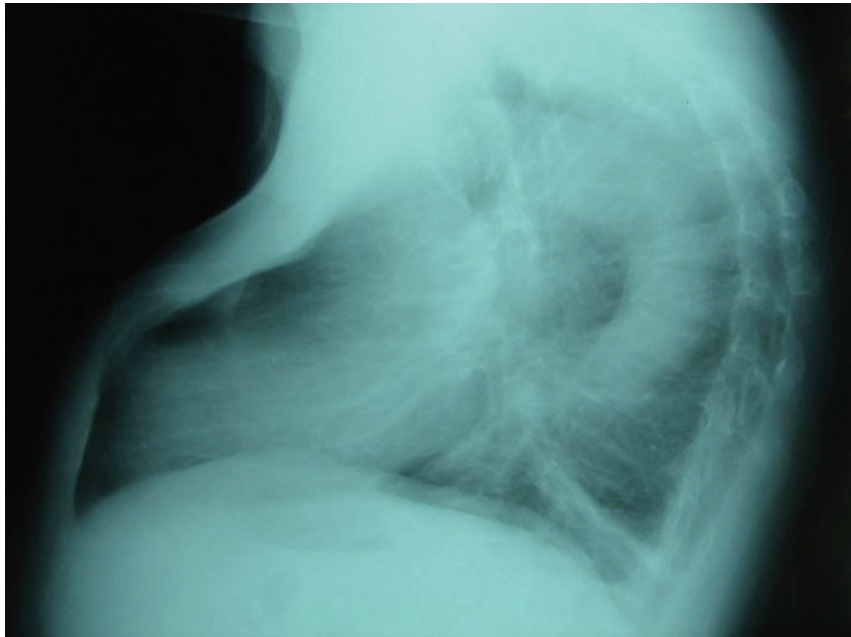

Figure 2. Chest X-ray revealed marked thoracic kyphosis.

He was healthy until 5 years before admission and had no family history of metabolic bone disease. On physical examination, the height was $160 \mathrm{~cm}$ and the weight was 60 $\mathrm{kg}$. He had pectus carinatum without a limb deformity (Fig. 1). Plain radiograph revealed marked thoracic kyphosis (Fig. 2) and pseudofracture in the right femoral neck (Fig. 3). Laboratory findings are shown in Table 1. A normal level of serum calcium, elevated level of serum alkaline phosphatase (ALP), and inappropriately increased urinary phosphate excretion despite extreme hypophosphatemia were noted. Endocrine examination showed a normal level of serum parathyroid hormone $(\mathrm{PTH})$, calcitonin and inappropriately low level of 1,25-dihydroxyvitamin D. Serum FGF23, which was measured by the sandwich enzyme-linked immunosorbent assay (ELISA) at the Department of Nephrology and Endocrinology of the University of Tokyo Hospital with the patient's consent (2), was in the upper limit of normal. Arterial blood gas analysis showed no metabolic acidosis and the result of urinalysis indicated the absence of diffuse tubular impairment. He was diagnosed as adult-onset hypophosphatemic osteomalacia caused by renal phosphate wasting associated with FGF23. Exhaustive investigation including careful physical examination, MRI of the head, neck and extremities, CT of the chest and abdomen, and serum tumor markers including serum prostate-specific antigen

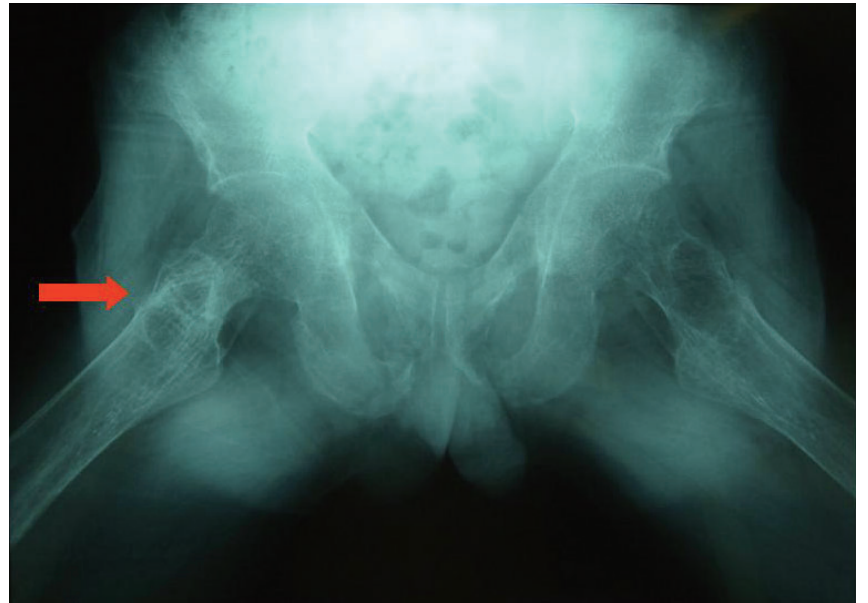

Figure 3. Hip X-ray revealed pseudofracture in the right femoral neck.

identified no evidence of a neoplastic lesion potentially responsible for TIO. The clinical course after admission is shown in Fig. 4. Even after two-week oral administration of neutral phosphate of $1.5 \mathrm{~g}$ per day together with activated vitamin $\mathrm{D}$ of $4 \mu \mathrm{g}$ per day, serum phosphate remained low with a further increase in urinary phosphate excretion. Within a week after initiation of additional oral administration of dipyridamole of $150 \mathrm{mg}$ per day, serum phosphate maintained the level of over $2 \mathrm{mg} / \mathrm{dl}$ with increased percent tubular reabsorption of phosphate (\%TRP). Bilateral diffuse leg pain and proximal muscle weakness showed signs of recovering within 6 weeks after initiation of therapy and were almost completely relieved within 15 weeks, and he became fully ambulatory.

\section{Discussion}

FGF23 has been recognized as a hormonal factor regulating phosphate and vitamin D metabolism since FGF23 was reported to be abundantly expressed in a patient with hypophosphatemic osteomalacia (3). FGF23 suppresses phosphate reabsorption in proximal renal tubular cells. In addition, it reduces serum 1,25-dihydroxyvitamin $\mathrm{D}$ level by mediating the several enzymes associated with vitamin D metabolism (4). Serum FGF23 may respond to serum phosphate in a 
Table 1. Results of Laboratory Tests on Admission

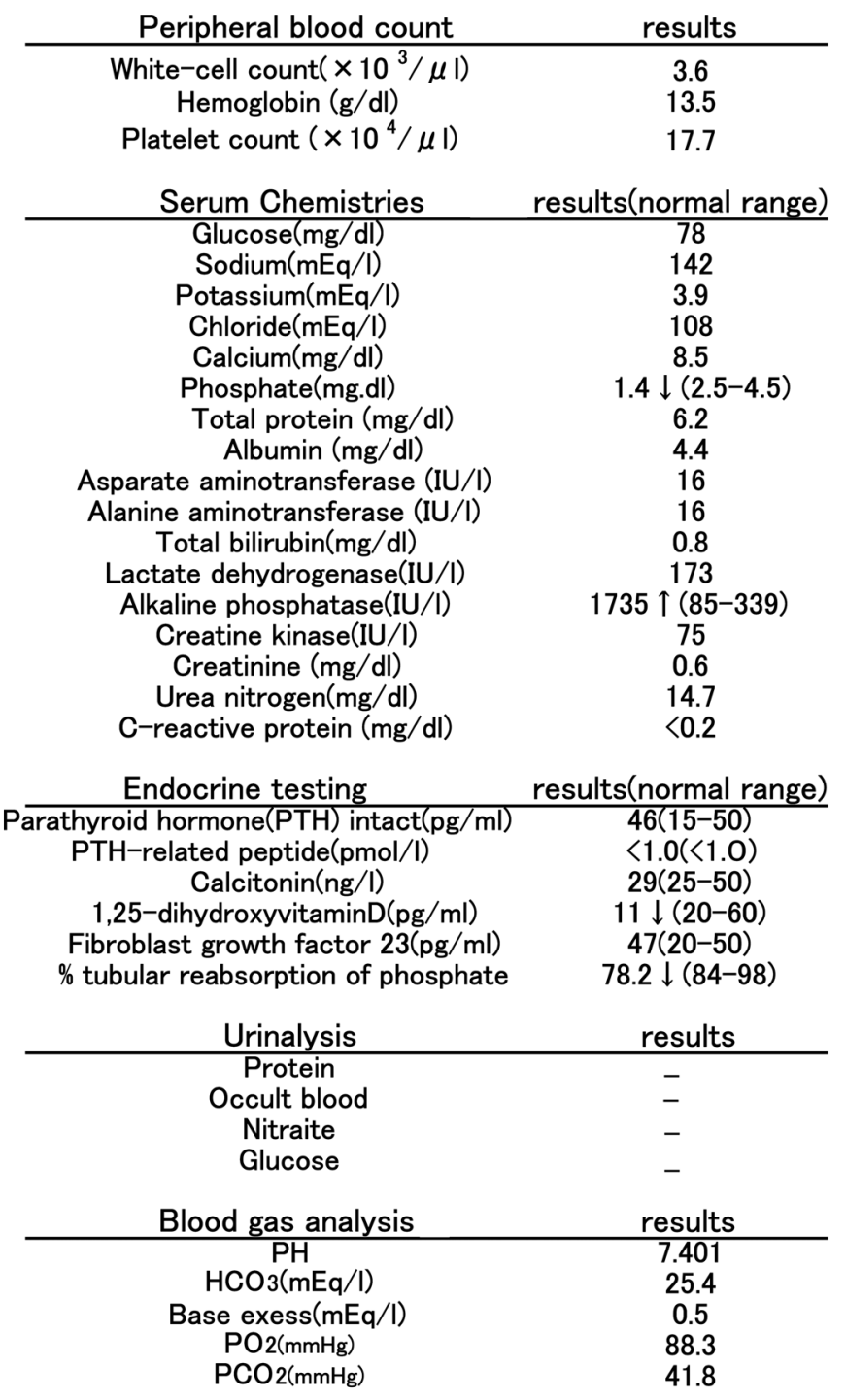

feedback system (5). In the present case, given that serum FGF23 was inappropriately high with a low level of serum 1,25-dihydroxyvitamin D despite extreme hypophosphatemia, osteomalacia due to excessive action of FGF23 was the most likely cause.

Although the genetic test has not yet been performed, hereditary hypophosphatemic osteomalacia diseases associated with FGF23 to be differentiated from the present case include XLH accounting for 80 percent of familial phosphate wasting (6), although rare, ADHR and ARHR. In acquired cases, TIO should be first considered in the differential diagnosis. In ADHR, serum FGF23 is elevated because mutations in a responsible gene called FGF23 (fibroblastic growth factor 23) enhance the expression or suppress the degradation of FGF23, whereas in TIO, a responsible tumor overproduces FGF23. The genes responsible for XLH and ARHR were also identified and termed PHEX (phosphateregulating gene with homologies to endopeptidases on the $X$-chromosome) and DMPI (dentin matrix protein 1), respectively $(7,8)$. But the relation between these genes and ele- vated serum FGF23 has not yet been clarified. Over 20 percent of XLH are believed to develop in a sporadic manner (9) and then are inherited in an X-linked dominant manner (10). In XLH, muscle weakness seen in other causes of osteomalacia is typically absent (11) and enthesopathy, which is bone proliferation at the sites of ligament, tendon and joint capsule, is a common finding, particularly in adults (11). Clinical manifestation of the present case is inconsistent with XLH because he had marked proximal muscle weakness and no radiographic finding of enthesopathy. Further, manifestation of rickets is absent in his daughter, which deems an X-linked dominant disorder unlikely. In ADHR, the levels of serum FGF23 or the onset varies widely because ADHR is incompletely penetrant. Consequently, patients often develop symptoms after puberty and delayed onset over 40 years old has been reported (12). If the present case would be sporadic ADHR, his two children could develop osteomalacia in the future. ARHR is the most uncommon hereditary hypophosphatemic osteomalacia associated with FGF23. Most of the reported ARHR cases developed during childhood unlike the present case. Even adult ARHR cases at ages over 30 years old had reportedly clinical problems with their legs during childhood (8). Thus, differentiation from TIO, as being an acquired phenotype, is the most important. In TIO, the level of FGF23 is inappropriately elevated (13) or near the upper limit of normal (14) despite extreme hypophosphatemia and tumors responsible for this disorder are characteristically benign, of mesenchymal origin (3) and prostate or oat cell carcinoma rarely contributes to this disease $(15,16)$. In the present case, no neoplstic lesion potentially responsible for this disease was identified in a thorough search including imaging studies of his entire body. However, the responsible tumor is often small and difficult to locate even on thorough clinical examination and often does not become evident for a long time after the onset of symptoms (17). Moreover, it has been pointed out that TIO is more common and may account for a substantial fraction of nonfamilial, adult-onset idiopathic hypophosphatemic osteomalacia (2), Thus, careful follow-up of surveillance for the tumor is required in the present case.

Drug therapy is based on phosphate supplements together with a large amount of activated vitamin $\mathrm{D}$, which is expected to relieve clinical symptoms and reduce the levels of serum ALP. The adverse effects of oral phosphate include gastrointestinal symptoms and hyperparathyroidism secondary to hypocalcemia, which aggravates renal phosphate wasting. Additional activated vitamin D may be helpful for prevention of hyperparathyroidism as well as supplementation with relatively low circulating 1,25-dihydroxyvitamin D levels. Adjustment of the dosage of activated vitamin D is necessary to avoid hypercalcemia or hypercalciuria. Dipyridamole has been reported to facilitate phosphate reabsorption in proximal renal tubular cells in adult with idiopathic hyperphosphaturia (18). In contrast, dipyridamole therapy has reportedly had no effect on serum phosphate or its urinary excretion in children with XLH (19). In the present 


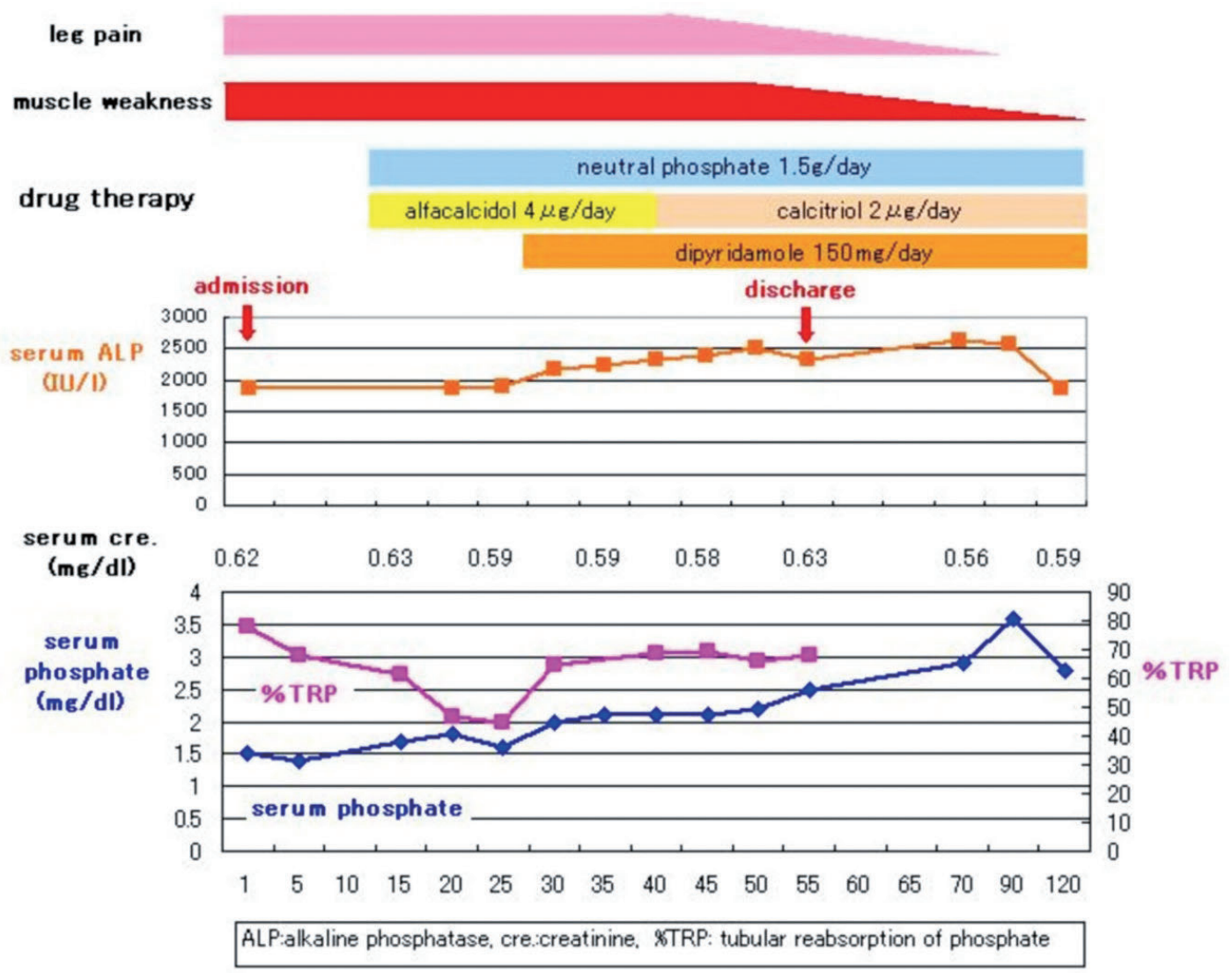

Figure 4. Clinical course after admission.

case, the administration of dipyridamole resulted in stabilized serum phosphate levels with increased \% TRP. Although some hypotheses of dipyridamole's effect on the renal tubules have been considered $(18,20)$, the mechanism of action on the renal tubules and the reason as to why its effect varies with individuals or age remain unknown.

When the present case was diagnosed as ankylosing spondylitis, he already presented with "unexplained" high levels of serum ALP. Unfortunately, hypophosphatemic bone disease was unsuspected by the primary physician because the patient did not have any bone deformities at the time. In general clinical practice, the measurement of serum phosphate is not routine. However, hypophosphatemic osteomalacia should be considered in the differential diagnosis of unexplained bone pain or muscle weakness even if bone deformity is absent.

\section{References}

1. Ryan EA, Reiss E. Oncogenous osteomalacia. Review of the world literature of 42 cases and report of two new cases. Am J Med 77: 501-512, 1984.

2. Yamazaki Y, Okazaki R, Shibata M, et al. Increased circulatory level of biologically active full-length FGF-23 in patients with hypophosphatemic rickets/osteomalacia. J Clin Endocrinol Metab 87: 4957-4960, 2002.

3. John MR, Wickert H, Zaar K, et al. A case of neuroendocrine oncogenic osteomalacia associated with a PHEX and fibroblast growth factor-23 expressing sinusidal malignant schwannoma. Bone 29: 393-402, 2001.

4. Fukumoto S, Yamashita T. FGF23 is a hormone-regulating phosphate metabolism-unique biological characteristics of FGF23. Bone 40: 1190-1195, 2007.

5. Gupta A, Winer K, Econs MJ, Marx SJ, Collins MT. FGF-23 is elevated by chronic hyperphosphatemia. J Clin Endocrinol Metab 89: 4489-4492, 2004.

6. Jan de Beur SM, Levine MA. Molecular pathogenesis of hypophosphatemic rickets. J Clin Endocrinol Metab 87: 2467-2473, 2002.
7. The HYP Consortium. A gene (PEX) with homologies to endopeptidases is mutated in patients with $\mathrm{X}$-linked hypophosphatemic rickets. Nat Ganet 11: 130-136, 1995.

8. Lorenz-Depiereux B, Bastepe $M$, Benet-Pagès A, et al. DMP1 mutations in autosomal recessive hypophosphatemia implicate a bone matrix protein in the regulation of phosphate homeostasis. Nat Genet 38: 1248-1250, 2006.

9. Dixon PH, Christie PT, Wooding C, et al. Mutational analysis of PHEX gene in X-linked hypophosphatemia. J Clin Endocrinol Metab 83: 3615-3623, 1998.

10. Holm IA, Nelson AE, Robinson BG, et al. Mutational analysis and genotype-phenotype correlation of the PHEX gene in Xlinked hypophosphatemic rickets. J Clin Endocrinol Metab 86: 3889-3899, 2001.

11. Jacobson JA, Kalume-Brigido M. Case 97: X-linked hypophosphatemic osteomalacia with insufficiency fracture. Radiology $\mathbf{2 4 0}$ : 607-610, 2006.

12. Econs MJ, McEnery PT. Autosomal dominant hypophosphatemic rickets/osteomalacia: clinical characterization of a novel renal phosphate-wasting disorder. J Clin Endocrinol Metab 82: 674-681, 
1997.

13. Jonsson KB, Zahradnik R, Larsson $\mathrm{T}$, et al. Fibroblast growth factor 23 in oncogenic osteomalacia and X-linked hypophosphatemia. N Engl J Med 348: 1656-1663, 2003.

14. Yoshioka K, Nagata R, Ueda M, et al. Phosphaturic mesenchymal tumor with symptoms related to osteomalacia that appeared one year after tumorectomy. Intern Med 45: 1157-1160, 2006.

15. Lyles KW, Berry WR, Haussler M, Harrelson JM, Drezner MK Hypophosphatemic osteomalacia: association with prostatic carcinoma. Ann Intern Med 93: 275-278, 1980.

16. Taylor HC, Fallon MD, Velasco ME. Oncogenic osteomalacia and inappropriate antidiuretic hormone secretion due to oat-cell carcinoma. Ann Intern Med 101: 786-788, 1984.

17. Clunie GP, Fox PE, Stamp TC. Four cases of acquired hypophos- phataemic ('oncogenic') osteomalacia. Problems of diagnosis, treatment and long-term management. Rheumatology (Oxford) 39: 1415-1421, 2000.

18. Prie D, Blanchet FB, Essig M, Jourdain JP, Friedlander G. Dipyridamole decreases renal phosphate leak and augments serum phosphorus in patients with low renal phosphate threshold. J Am Soc Nephrol 9: 1264-1269, 1998.

19. Seikaly MG, Quigley R, Baum M. Effect of dipyridamole on serum and urinary phosphate in X-linked hypophosphatemia. Pediatr Nephrol 15: 57-59, 2000.

20. Prie D, Couette S, Fernandes I, Silve C, Friedlander G. Pglycoprotein inhibitors stimulate renal phosphate reabsorption in rats. Kidney Int 60: 1069-1076, 2001.

(C) 2008 The Japanese Society of Internal Medicine http://www.naika.or.jp/imindex.html 